\title{
Influence de l'ordre d'introduction des solutés (Naphtol-2 et Calcium) sur l'adsorption des tensioactifs sur charbon actif
} F400

\section{The effect of the introduction order of the solutes(Napthol-2 and Calcium) on the adsorption of surfactants onto powder activated carbon F400}

\section{S. Souabi, A. Yaacoubi et M. Mazet}

Volume 7, numéro 1, 1994

URI : https://id.erudit.org/iderudit/705188ar

DOI : https://doi.org/10.7202/705188ar

Aller au sommaire du numéro

\section{Éditeur(s)}

Université du Québec - INRS-Eau, Terre et Environnement (INRS-ETE)

ISSN

0992-7158 (imprimé)

1718-8598 (numérique)

Découvrir la revue

Citer cet article

Souabi, S., Yaacoubi, A. \& Mazet, M. (1994). Influence de l'ordre d'introduction des solutés (Naphtol-2 et Calcium) sur l'adsorption des tensioactifs sur charbon actif F400. Revue des sciences de l'eau / Journal of Water Science, 7(1), 43-54. https://doi.org/10.7202/705188ar

\section{Résumé de l'article}

L'effet de l'ordre d'introduction des solutés Naphtol-2 et Calcium (N-2 et Ca) sur l'adsorption de tensioactifs par le charbon actif en poudre (CAP) F400 brut a été étudié en discontinu. Les mélanges étudiés sont: le tensioactif anionique dodécyl-sulfate de sodium (DSS) et le N-2; le DSS, le N-2 et le Ca; le tensioactif cationique bromure de benzyldodécyldiméthylammonium (BBDDA) et le N-2 et enfin le BBDDA et $\mathrm{Ca}$. Les résultats expérimentaux montrent tout d'abord que quel que soit l'ordre d'introduction des solutés $\mathrm{N}-2$ et $\mathrm{Ca}$, les concentrations en DSS à l'équilibre sont sensiblement identiques. Par ailleurs, la cinétique d'adsorption du DSS et du BBDDA croît en présence des ions Ca.

Cependant, la cinétique d'adsorption du N-2 en présence du DSS diminue ce qui se traduit par une inhibition de l'adsorption du N-2 par le DSS.

Quant à l'adsorption du BBDDA en présence du N-2 pour différents ordres d'introduction, le tracé des isothermes de LANGMUIR montre que la capacité maximale d'adsorption $\Gamma \infty$ du BBDDA ne change pas tandis que la constante de LANGMUIR (K) diminue. Par contre la capacité maximale d'adsorption du N-2

** varie légèrement et passe de 1,67 à $1,5 \mathrm{mmol} / \mathrm{g}$ pour différent ordre d'introduction du BBDDA. On note également que $\Gamma \infty$ du N-2 en présence de concentrations croissantes en BBDDA diminue fortement et passe de 1,67 à 0,8 $\mathrm{mmol} / \mathrm{g}$.

L'ajout de N-2 après 3 heures de contact du BBDDA/CAP ne provoque pas la désorption de ce dernier. Le même comportement a été observé avec le $\mathrm{N}-2$, alors que l'ajout du DSS au mélange N-2/CAP provoque la désorption de celui ci. 


\title{
Influence de l'ordre d'introduction des solutés (Naphtol-2 et Calcium) sur l'adsorption des tensioactifs sur charbon actif F400
}

\author{
The effect of the introduction order of the solutes \\ (Napthol-2 and Calcium) on the adsorption \\ of surfactants onto powder activated carbon F400
}

S. SOUABI', A. YAACOUBI2 et M. MAZET ${ }^{3}$

Reçu le 14 mai 1993, accepté le 10 novembre $1993^{*}$.

\section{SUMMARY}

\begin{abstract}
The effect of the introduction onder of naphtol-2 and calcium (N-2 and $\mathrm{Ca}$ ) on the adsorption of surfactants by raw powdered activated carbon (PAC) F 400 has been studied in a static system. Studied mixtures included the anionic surfactant: sodium dodecyl-sulfate (SDS) with $\mathrm{N}-2$ or with $\mathrm{N}-2+\mathrm{Ca}$, and the cationic surfactant benzyldodecyldimethylammonium bromide (BDDAB) with N-2, or with $\mathrm{Ca}$. The experimental results demonstrate that whatever the order of solute introduction, the SDS concentrations at equilibrium are almost identical. Moreover, the adsorption kinetics of SDS and BDDAB increase in the presence of calcium ions. The adsorption kinetics of $\mathrm{N}-2$ decrease in the presence of SDS, suggesting inhibition of $\mathrm{N}-2$ adsorption by SDS. Concerning the adsorption of BDDAB in the presence of $\mathrm{N}-2$, for different introduction orders, plotting of the LANGMUIR isotherms shows that the maximal BDDAB adsorption capacity $\Gamma^{\infty}$, does not change but the LANGMUIR constant (K) decreases. For naphtol-2, however the maximal adsorption capacity $\Gamma^{\infty}$, does vary slightly, going from 1.67 to $1.5 \mathrm{mmol} / \mathrm{g}$ for different BDDAB introduction orders. In the presence of increasing BDDAB concentrations, the maximum adsorption capacity of $\mathrm{N}-2$ decreases from 1.67 to $0.8 \mathrm{mmol} / \mathrm{g}$.
\end{abstract}

The addition of N-2 after 3 hours of BDDAB/PAC contact does not lead to BDDAB desorption. Similar behavior is observed on addition of $\mathbf{N - 2}$ after 3 hours of SDS/PAC contact, even though the addition of SDS to a N-2/PAC mixture leads to $\mathrm{N}-2$ desorption.

Key-words : adsorption, activated carbon, surfactants, co-adsorption, naphtol-2, calcium.

1. Faculté des Sciences MEKNES (MAROC)

2. Faculté des Sciences SEMLALIA MARRAKECH (MAROC)

3. Faculté des Sciences Laboratoire de génie chimique Traitement des eaux LIMOGES (FRANCE)

- Les commentaires seront reçus jusqu'au 31 octobre 1994. 


\section{RÉSUMÉ}

L'effet de l'ordre d'introduction des solutés Naphtol-2 et Calcium (N-2 et Ca) sur l'adsorption de tensioactifs par le charbon actif en poudre (CAP) F400 brut a été étudié en discontinu. Les mélanges étudiés sont : le tensioactif anionique dodécyl-sulfate de sodium (DSS) et le $\mathrm{N}-2$; le DSS, le $\mathrm{N}-2$ et le Ca ; le tensioactif cationique bromure de benzyldodécyldiméthylammonium (BBDDA) et le N-2 et enfin le BBDDA et $\mathrm{Ca}$. Les résultats expérimentaux montrent tout d'abord que quel que soit l'ordre d'introduction des solutés $\mathbf{N}-2$ et $\mathrm{Ca}$, les concentrations en DSS à l'équilibre sont sensiblement identiques. Par ailleurs, la cinétique d'adsorption du DSS et du BBDDA crô̂t en présence des ions Ca. Cependant, la cinétique d'adsorption du $\mathrm{N}-2$ en présence du DSS diminue ce qui se traduit par une inhibition de l'adsorption du N-2 par le DSS.

Quant à l'adsorption du BBDDA en présence du N-2 pour différents ordres d'introduction, le tracé des isothermes de LANGMUIR montre que la capacité maximale d'adsorption $\Gamma^{\circ}$ du BBDDA ne change pas tandis que la constante de LANGMUIR (K) diminue. Par contre la capacité maximale d'adsorption du N-2 $\Gamma^{\infty}$ varie légèrement et passe de 1,67 à $1,5 \mathrm{mmol} / \mathrm{g}$ pour différent ordre d'introduction du BBDDA. On note également que $\Gamma^{\infty} \mathrm{du} N$-2 en présence de concentrations croissantes en BBDDA diminue fortement et passe de 1,67 à $0,8 \mathrm{mmol} / \mathrm{g}$.

L'ajout de N-2 après 3 heures de contact du BBDDA /CAP ne provoque pas la désorption de ce dernier. Le même comportenent a été observé avec le $\mathrm{N}-2$, alors que l'ajout du DSS au mélange N-2/CAP provoque la désorption de celui-ci.

Mots clés : adsorption, charbon actif, tensioactifs, co-adsorption naphtol-2, calcium.

\section{1 - INTRODUCTION}

La pollution des eaux par de nombreux composés souvent toxiques et difficilement biodégradables, nécessite fréquemment l'utilisation du charbon actif comme matériau adsorbant dans les usines de préparation de l'eau potable ou industrielle. De nombreuses études effectuées par DORE et al., (1982), LE CLOIREC et MARTIN (1984), MAZET et al., (1988), PREVOST et al., (1990), ont montré que le charbon actif est efficace pour éliminer différents polluants organiques dans une eau à traiter.

Un micropolluant étant rarement seul en solution, il est plus intéressant de connaître les modifications de ses propres paramètres d'équilibre d'adsorption lorsqu'il se trouve en présence d'autres co-adsorbats.

La sélectivité d'adsorption des composés organiques par le charbon actif dépend des affinités intrinsèques de chaque couple GAID et al., (1982), LAFRANCE et al., (1985), mais également de l'influence des co-adsorbats sur la surface d'adsorption. De plus les interactions entre les adsorbats à l'état libre ou fixé peuvent conditionner la nature et la spécificité d'adsorption par le charbon actif dans le traitement des eaux à potabiliser dont la qualité peut varier en fonction du temps. 
Dans ce travail nous nous intéressons à l'étude des paramètres d'équilibre d'adsorption du BBDDA et du DSS par F400 saturé en N-2 et également des paramètres d'équilibre d'adsorption du N-2 par F400 saturé en DSS ou en BBDDA.

Nous avons déterminé linfluence des ions calcium car ce dernier se trouve en quantité abondante dans l'eau et en proportions significatives à la surface du charbon.

Les résultats expérimentaux peuvent contribuer à la compréhension des interactions possibles entre les différents polluants organiques à éliminer par adsorption sur le CAP et prédire leur fixation dans le cas d'une adsorption multiple tout en faisant intervenir l'effet de l'ordre d'introduction des solutés.

\section{2 - MATÉRIELS ET MÉTHODES}

\subsection{Matériau utilisé}

Le charbon actif en poudre (CAP) utilisé provient d'un charbon granulé $(0,4$ et $1,7 \mathrm{~mm}$ ) Filtrasorb-400 de Chemviron, broyé, tamisé pour obtenir une granulométrie inférieure ou égale à $50 \mu \mathrm{m}$; la surface spécifique déterminée par BET pour une granulométrie de 0,4 et $1,7 \mathrm{~mm}$ est voisine de $1200 \mathrm{~m} 2 / \mathrm{g}$.

Le tensioactif utilisé est le bromure de benzyldodécyldiméthylammonium (BBDDA) $\mathrm{CH}_{3}\left(\mathrm{CH}_{2}\right)_{11}\left(\mathrm{CH}_{3}\right)_{2} \mathrm{NCH}_{2} \mathrm{C}_{6} \mathrm{H}_{5} \mathrm{Br}$. II a été synthétisé selon la méthode utilisée par (SHELTON et al., 1948).

Le $\mathrm{N}-2$ est de formule $\mathrm{C}_{10} \mathrm{H}_{8} \mathrm{O}$, le dodécyl sulfate de sodium est de formule $\mathrm{CH}_{3}\left(\mathrm{CH}_{2}\right)_{11} \mathrm{OSO}_{3} \mathrm{Na}$. La pureté de ces produits est supérieure à $98 \%$.

\subsection{Méthodes analytiques}

Les tensioactifs ont été dosés seion les méthodes décrites par DUSART et al., (1988). Le N-2 a été dosé par spectrophotométrie dans l'ultraviolet en utilisant un appareil BAUCH et LOMB spectronic 21, à une longueur d'onde de $220 \mathrm{~nm}$. La limite de détection est de $100 \mu \mathrm{g} / \mathrm{l}$.

Aucune interférence significative dans le dosage en UV des solutions de $\mathrm{N}$-2 de concentrations comprises entre $t$ à $2 \mathrm{mg} / \mathrm{l}$ n'a été observé en présence de DSS ou de BBDDA et/ou des ions calcium.

\section{3 Étude de l'adsorption en flacon}

L'étude de la cinétique d'adsorption est réalisée pour une masse de CAP constante dans un litre d'eau distillée $(5,5<\mathrm{pH}<6$.)

Après agitation à l'abri de la lumière au bout d'un temps déterminé, et filtration sur membrane $(0,45 \mu \mathrm{m})$, la concentration est alors déterminée par les méthodes précédemment décrites. 
L'étude de la cinétique permet d'évaluer le temps nécessaire pour atteindre un pseudo-équilibre. Ce temps est évalué à 3 heures pour un composé seul BBDDA, DSS, ou $\mathrm{N}-2$ ainsi que dans le cas des mélanges et sera retenu pour estimer les paramètres d'équilibre d'adsorption.

\section{3 - RÉSULTATS ET DISCUSSIONS}

3.1 Influence de l'ordre d'introduction des solutés sur les cinétiques rapides d'adsorption dans le système (DSS + $\mathrm{N}-2$ ) et (BBDDA + $\mathrm{Ca}$ )

L'ordre d'introduction des composés et du CAP est réalisé suivant les séquences du tableau 1.

Tableau 1 Ordre d'introduction des solutés (DSS $+\mathrm{N}-2$ ) sur le CAP en suspension dans l'eau.

Table 1 Introduction order of the solutes $(S D S+N-2)$ on PAC in water suspension.

\begin{tabular}{|lcccc|}
\hline Mélange initial & Temps de contact h & Ajout & Temps de contact h & Courbe \\
\hline DSS + CAP & Variable & - & - & 1 \\
DSS + N-2 + CAP & Variable & - & - & 2 \\
DSS + CAP & 3 & N-2 & Variable & $1+3$ \\
N-2 + CAP & 3 & DSS & Variable & 4 \\
\hline
\end{tabular}

Les résultats expérimentaux de la cinétique d'adsorption du DSS en présence du N-2 sont représentés sur la figure 1 .

Les courbes (1) et (2) de la figure 1 montrent que la présence de $\mathrm{N}-2$ inhibe en partie l'adsorption du DSS lorsque le mélange (DSS + CAP) est en équilibre d'adsorption. L'ajout du N-2 provoque la désorption d'une fraction du DSS adsorbé (courbe 3), et le nouvel équilibre établi correspond à celui obtenu pour les mêmes concentrations des solutés lorsque ceux-ci sont introduits simultanément (courbe 2). Dans le cas ou DSS est introduit dans le mélange $(\mathrm{N}-2+\mathrm{CAP})$ à l'équilibre, la courbe 4 montre que l'inhibition est la même.

En conclusion, les courbes de la figure 1 indiquent que, quel que soit l'ordre d'introduction des solutés, on constate expérimentalement que les concentrations en DSS à l'équilibre sont sensiblement égales. 


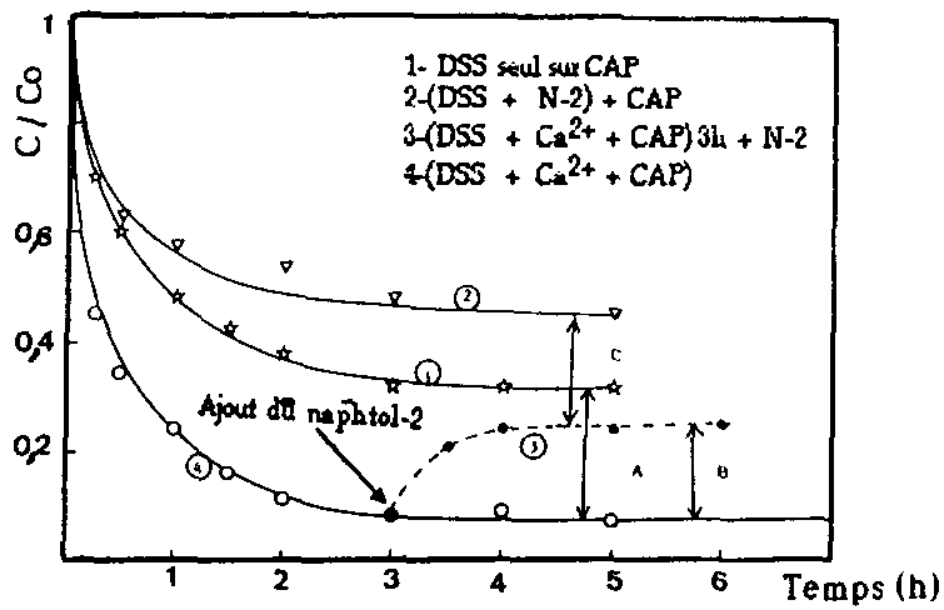

Figure 1 Cinétique d'adsorption du DSS en présence du naphtol-2 et des ions calcium. Effet de l'ordre d'introduction des solutés.

Adsorption Kinetic of SDS in the presence of $\mathrm{N}-2$ and calcium ions. The solutes introduction order effect.

Les résultats des dosages du N-2 pour un ordre d'introduction des solutés similaire aux séquences du tableau (1) sont représentés sur la figure 2, les mêmes remarques sont formulées avec le $\mathrm{N}-2$ lequel voit en partie son adsorption inhibée par le DSS (courbe 1 et 2) figure 2. Lorsque le DSS est ajouté au mélange (N-2/CAP) il provoque la désorption de celui-ci; par

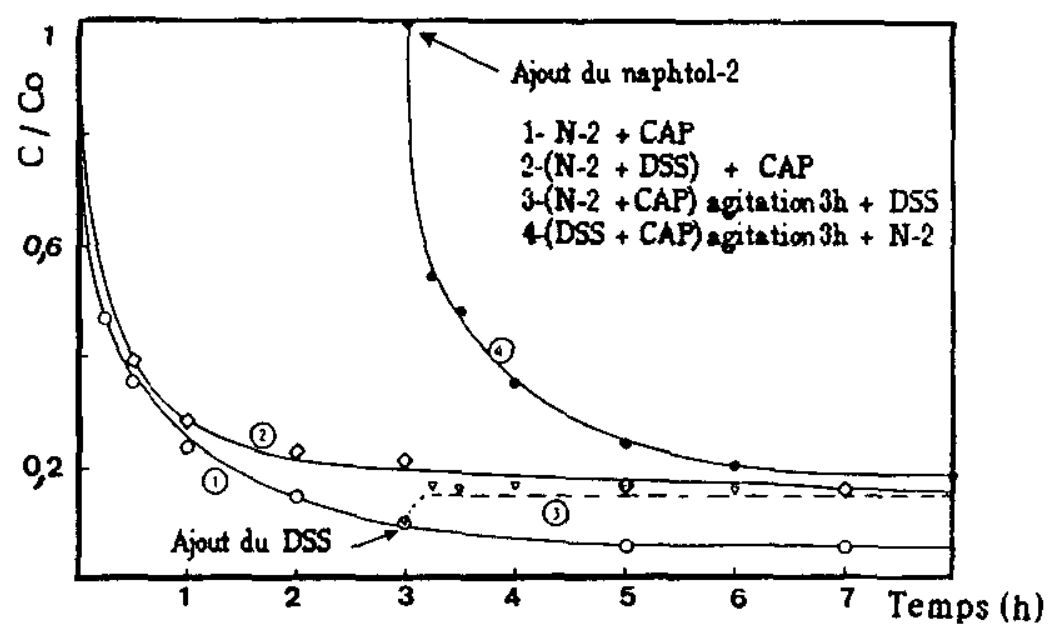

Figure 2 Cinétique d'adsorption du naphtol-2 en présence de DSS. CAP = $7 \mathrm{mg} / 1 ; \mathrm{C}_{\mathrm{N}-2}=1 \mathrm{mg} / 1 ; \mathrm{DSS} / \mathrm{N}-2=0,5 ; \mathrm{pH}=5,5$.

Adsorption Kinetic of $\mathrm{N}-2$ in the presence of $S D S$. CAP $=7 \mathrm{mg} / 1 ; C_{\mathrm{N}-2}=$ $1 \mathrm{mg} / \mathrm{i} ; \mathrm{DSS} / \mathrm{N}-2=0,5 ; \mathrm{pH}=5,5$. 
contre l'ajout du $\mathrm{N}-2$ au mélange DSS/CAP (courbe 4) montre que la concentration résiduelle en co-adsorbats est sensiblement identique à celle obtenue pour les courbes 2 et 3 (fig. 2). Quant à la cinétique d'adsorption du BBDDA sur F400 ( $\left.\mathrm{M}_{\mathrm{CAG}}=7 \mathrm{mg} / \mathrm{l}\right)$ en présence des ions calcium $\left(5,310^{-3}\right.$ mol/l), on remarque que l'ordre d'addition des ions calcium ne modifie pas la capacité maximale d'adsorption à l'équilibre du BBDDA.

Ainsi l'ajout de calcium au temps $t=3 \mathrm{~h}$ se traduit par une augmentation de l'adsorption du BBDDA atteignant ainsi le niveau obtenu lorsque le calcium est introduit simultanément avec le tensioactif cationique (BBDDA) (fig. 3).

En présence des ions calcium l'adsorption de DSS croît, ceci indique que la formation d'espèces telles que $\mathrm{DSCa}^{+},(\mathrm{DS})_{2} \mathrm{Ca}$, pourrait favoriser l'adsorption du DSS (sous ses formes libres ou associées) par interaction électrostatique avec des sites fonctionnels ionisés à la surface du charbon.

Ces résultats mettent en évidence le rôle joué par les ions calcium sur l'adsorption d'un composé organique anionique, le DSS, en favorisant l'approche de ce dernier à la surface du charbon. II faut noter également que la modification des propriétés électrostatiques de la surface du charbon en présence des ions calcium est responsable de l'augmentation de l'adsorption de BBDDA.

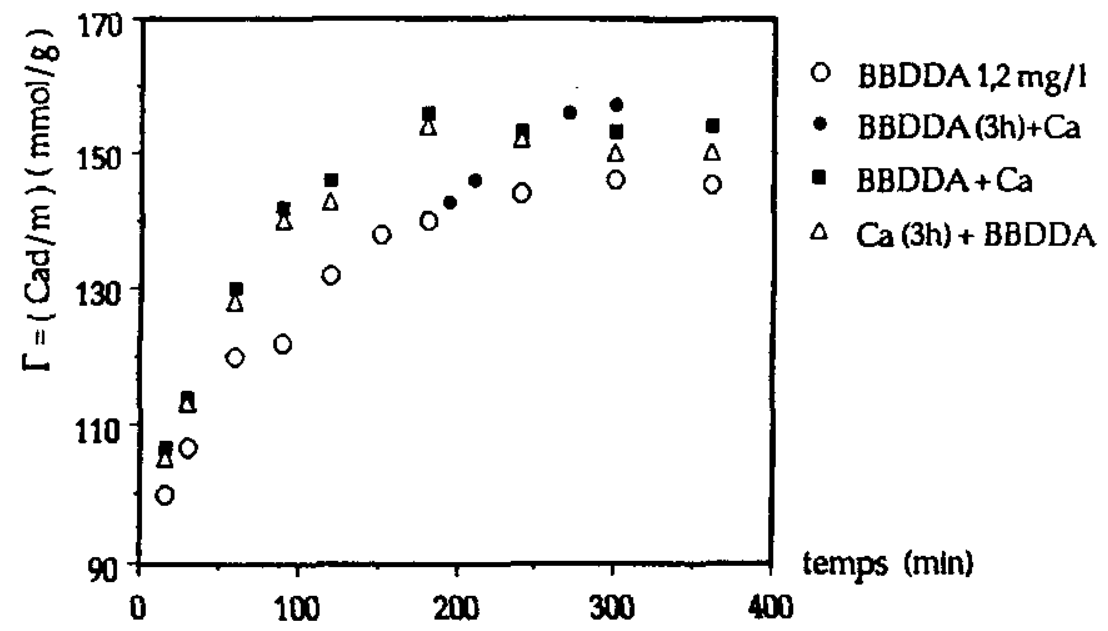

Figure 3 Influence de l'ordre d'introduction des ions calcium sur la cinétique d'adsorption du BBDDA. $\mathrm{C}_{\mathrm{BBDDA}}=3,12 \mu \mathrm{mol} / \mathrm{l}$.

Influence of calcium ions introduction order on the adsorption kinetic of $B D D A B . C_{\text {BDOAB }}=3,12 \mu \mathrm{molh}$.

\subsection{Influence de l'ordre d'introduction des solutés sur les para- mètres d'équilibre d'adsorption par F400 du BBDDA et $\mathrm{N}-2$}

Dans cette partie nous avons essayé de montrer l'influence de l'ordre dans lequel on ajoute les co-adsorbats à la susperision de charbon actif et d'étudier ainsi les modifications possibles des paramètres d'équilibre. 
L'ordre d'introduction des réactifs a été réalisé selon les séquences du tableau 2.

Tableau 2 Ordre d'introduction des solutés sur le CAP en suspension dans l'eau.

Table 2 Introduction order of the solutes $(B D D A B+N-2)$ on PAC in water suspension.

\begin{tabular}{|c|c|c|c|c|}
\hline Méłange initial & Temps de contact h & Ajout & Temps de contact $h$ & Dosage \\
\hline $\begin{array}{l}\text { BBDDA + CAP } \\
N-2+C A P \\
\text { BBDDA + N-2 + CAP } \\
N-2+C A P \\
\text { BBDDA + CAP }\end{array}$ & $\begin{array}{l}3 \\
3 \\
3 \\
3 \\
3\end{array}$ & $\begin{array}{c}- \\
- \\
- \\
\text { BBDDA } \\
\text { N-2 }\end{array}$ & $\begin{array}{l}- \\
- \\
- \\
3 \\
3\end{array}$ & $\begin{array}{c}\text { BBDDA } \\
\mathrm{N}-2 \\
\mathrm{~N}-2+\mathrm{BBDDA} \\
\mathrm{N}-2+\mathrm{BBDDA} \\
\mathrm{N}-2+\mathrm{BBDDA}\end{array}$ \\
\hline
\end{tabular}

Les transformées linéaires des isothermes de LANGMUIR pour le $\mathrm{N}-2$ $(1,2 \mathrm{mg} / \mathrm{l})$ ou $(8,3 \mu \mathrm{mol} / \mathrm{l})$ en présence du BBDDA $(3,12 \mu \mathrm{mol} / \mathrm{l})$ sont représentées sur la figure 4 .

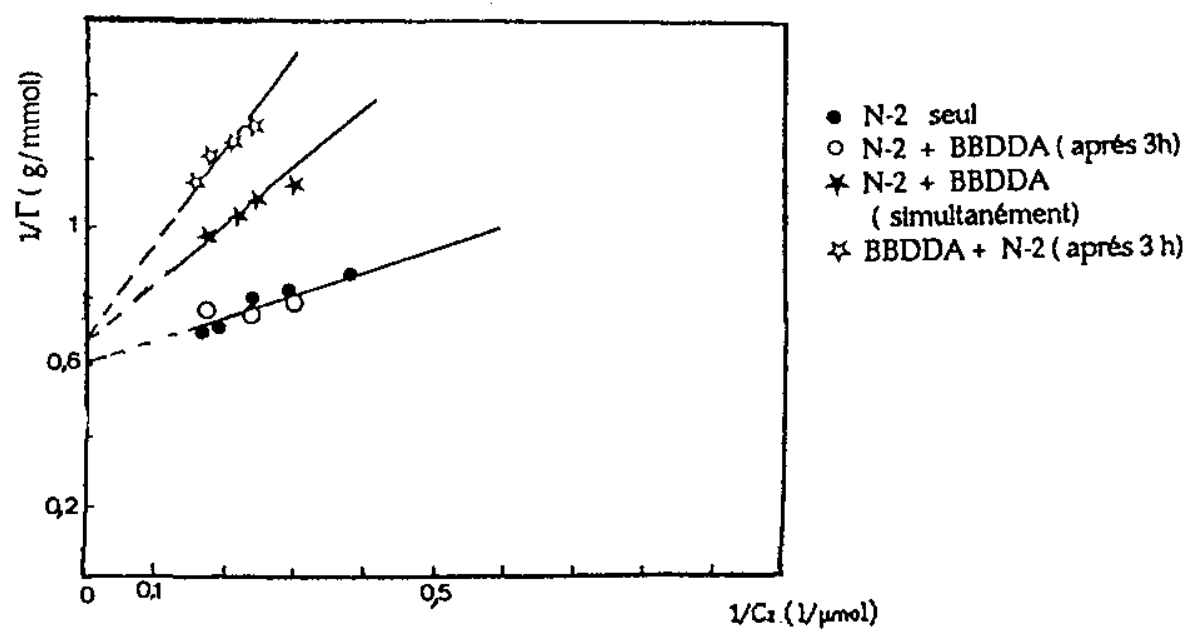

Figure 4 Dosage du N-2. $C_{\mathrm{N}-2}=8,3 \mu \mathrm{mol} / / ; \mathrm{C}_{\mathrm{BBDDA}}=3,12 \mu \mathrm{mol} / \mathrm{l}$.

Dosage of $N-2$. $C_{\mathrm{N}-2}=8,3 \mu \mathrm{mol} / \mathrm{i} ; C_{B D D B}=3,12 \mu \mathrm{mol} /$.

Par ailleurs, sur la figure 5, on représente l'adsorption du BBDDA $(3,12 \mu \mathrm{mol} / \mathrm{l})$ également en présence de $\mathrm{N}-2(8,3 \mu \mathrm{mol} / \mathrm{l})$.

Les paramètres d'équilibre (constante d'adsorption $\mathrm{K}$ et capacité maximale d'adsorption $\Gamma^{\infty}=A$ ) obtenus à partir des isothermes de LANGMUIR (cas du BBDDA et du N-2) sont indiqués sur le tableau 3. 


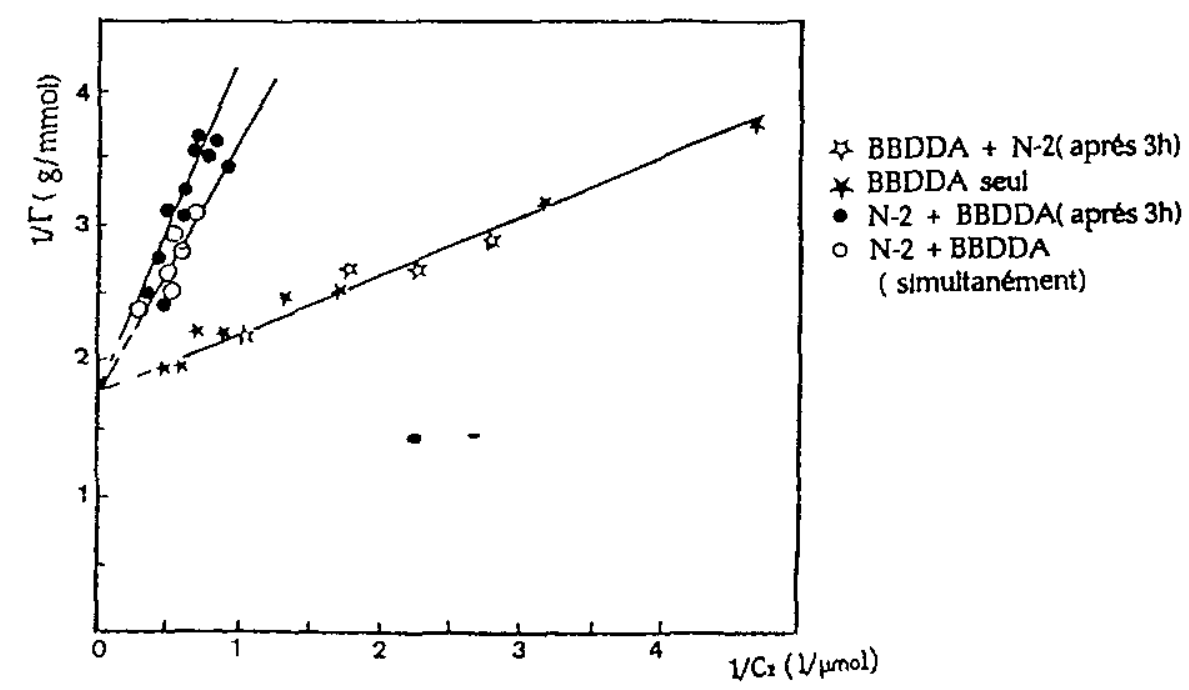

Figure 5 Dosage du BBDDA. $C_{\mathrm{N}-2}=8,3 \mu \mathrm{mol} ; \mathrm{C}_{\mathrm{BBDOA}}=3,12 \mu \mathrm{mol} / \mathrm{l}$.

Dosage of $B B D D A$. $C_{\mathrm{N} \cdot 2}=8,3 \mu \mathrm{mol} / \mathrm{l} ; C_{B D D A B}=3,12 \mu \mathrm{mol} / \mathrm{l}$.

Table $3 \quad$ Variation of the equilibrium parameters for the adsorption of DBBAB and $\mathrm{N}-2$ with respect to the inibitory $\mathrm{N}-2$ addition order.

$\Gamma^{\infty}=A=\mathrm{mmol} / \mathrm{g}$ (maximal adsorption capacity)

$B=K 106 \mathrm{Vmol}$ (LANGMUIR constant)

\begin{tabular}{|c|c|c|c|c|c|c|c|c|c|c|}
\hline & \multicolumn{2}{|c|}{$\begin{array}{c}\text { (N-2) } 1,2 \mathrm{mg} / \\
(8,3 \mu \mathrm{mol} / \mathrm{)})\end{array}$} & \multicolumn{2}{|c|}{$\begin{array}{c}\text { BBODA 1,2 mg/ } \\
(3,12 \mu \mathrm{mol} / \mathrm{l})\end{array}$} & \multicolumn{2}{|c|}{$\begin{array}{l}\mathrm{N}-2+(\mathrm{BBODA}) \\
\text { simultanément }\end{array}$} & \multicolumn{2}{|c|}{$\begin{array}{c}\mathrm{N}-2+(\mathrm{BBODA}) \\
\text { (après } 3 \mathrm{~h})\end{array}$} & \multicolumn{2}{|c|}{$\begin{array}{c}\text { BBODA + (N-2) } \\
\text { (après } 3 \mathrm{~h})\end{array}$} \\
\hline & A & B & A & B & A & B & A & B & A & B \\
\hline $\begin{array}{l}\text { Dosage } \\
\mathrm{N}-2\end{array}$ & 1,62 & 1,1 & - & - & 1,5 & 0,55 & 1,62 & 1,1 & 1,5 & 0,24 \\
\hline $\begin{array}{l}\text { Dosage } \\
\text { BBDDA }\end{array}$ & - & - & 0,59 & 4,9 & 0,59 & 0,95 & 0,59 & 0,7 & 0,59 & 4,9 \\
\hline
\end{tabular}

Pour avoir des données supplémentaires sur la nature des interactions physico-chimiques que peuvent avoir les composés BBDDA et $\mathrm{N}-2$ avec la surface du CAP en faisant varier l'ordre d'introduction des solutés, on a étudié l'évolution des paramètres d'équilibre d'adsorption du $\mathrm{N}-2$ en présence de concentrations croissantes en BBDDA (fig. 6). Par ailleurs, la capacité ultime d'adsorption $\Gamma^{\infty}$ du $\mathrm{N}-2$ diminue et passe de 1,67 à $0,8 \mathrm{mmol} / \mathrm{l}$ (figure 6). Dans notre cas la différence de capacité (entre le BBDDA seul=0,59 $\mathrm{mmol} / \mathrm{g}$ et le $\mathrm{N}-2$ seul $=1,67 \mathrm{mmol} / \mathrm{g}$ ) de $1,1 \mathrm{mmol} / \mathrm{g}$ serait en relation avec le nombre de sites sur lesquels le $\mathrm{N}-2$ se fixerait sans compétition. La constante $\mathrm{K}$ diminue légèrement puis croît pour tendre vers l'infini à partir d'une concentration d'environ $2 \mathrm{mg} / \mathrm{l}$ en BBDDA (fig. 6). Le rapport molaire BBDDA/N-2 étant de 0,6 . 


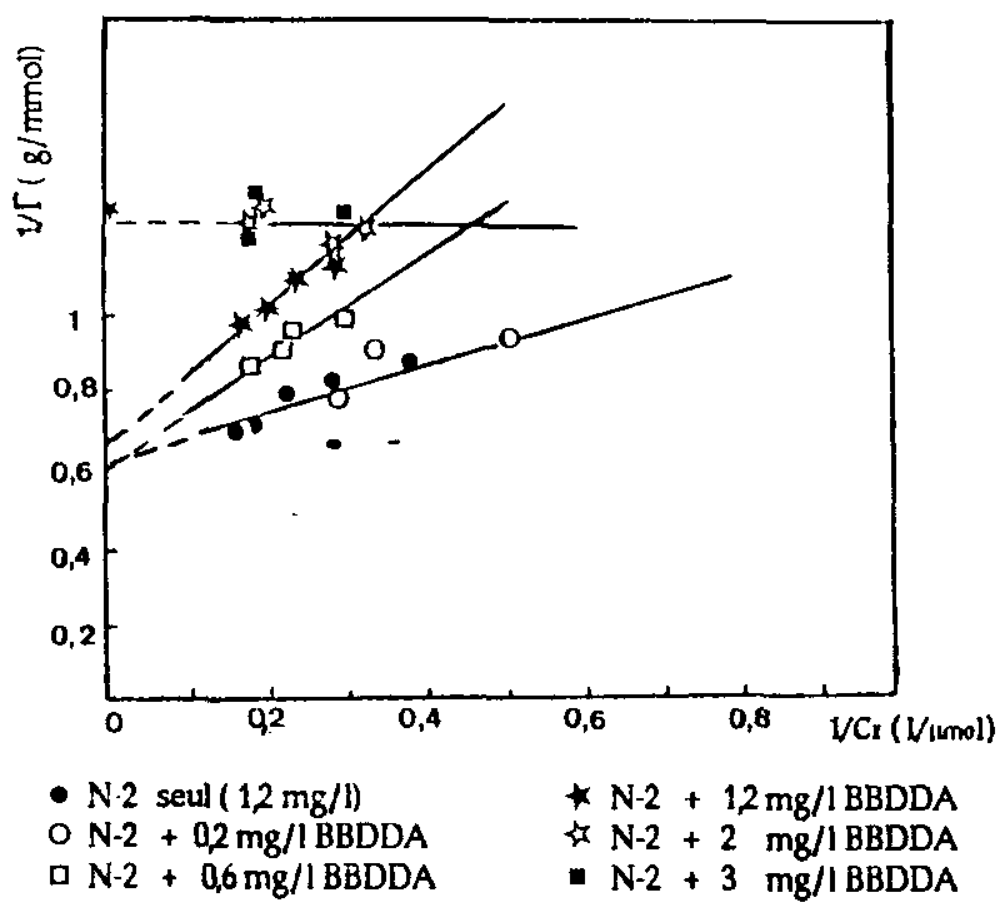

Figure 6 Influence du BBDDA sur l'adsorption du napthol-2 sur F400. $B D D A B$ influence on $\mathrm{N}-2$ adsorption onto PAC F400.

Ceci confirme que la surface du CAP est saturée en BBDDA. Un tel phénomène a été également observẻ au cours de l'étude de l'adsorption de NP8 en présence de DSS (DUSART et al., 1985). Ces auteurs montrent que la capacité maximale théorique d'adsorption du DSS en présence de NP8 est plus fortement diminuée que dans le cas inverse. On observe ainsi que la capacité d'adsorption de DSS en présence de concentrations croissantes sur le N-2 reste inchangée (fig. 7). Le même phénomène a été observé au cours de l'adsorption du BBDDA $(3,12 \mu \mathrm{mol} / \mathrm{l})$ en présence de concentrations variables en $\mathrm{N}-2$ (fig. 8 ).

L'évolution des paramètres d'équilibre suggère que la modification du nombre de sites du CAP occupés par le $\mathrm{N}-2$ pourrait être due à une modification de l'affinité du couple N-2/CAP lors de l'adsorption du BBDDA. La diminution de l'adsorption d'un composé aromatique comme le $\mathrm{N}-2$ en présence de concentrations importantes d'un co-adsorbat BBDDA pourrait être attribuée, d'une part, à l'encombrement moléculaire résultant de la présence du BBDDA qui empêche la diffusion intraparticulaire du $\mathrm{N}-2$ et, d'autre part, à.une inhibition probable due à une compétition d'adsorption.

Ainsi la stabilité du complexe de transfert de charge entre les noyaux aromatiques du CAP/N-2 serait diminuée par l'adsorption de la molécule de BBDDA. 


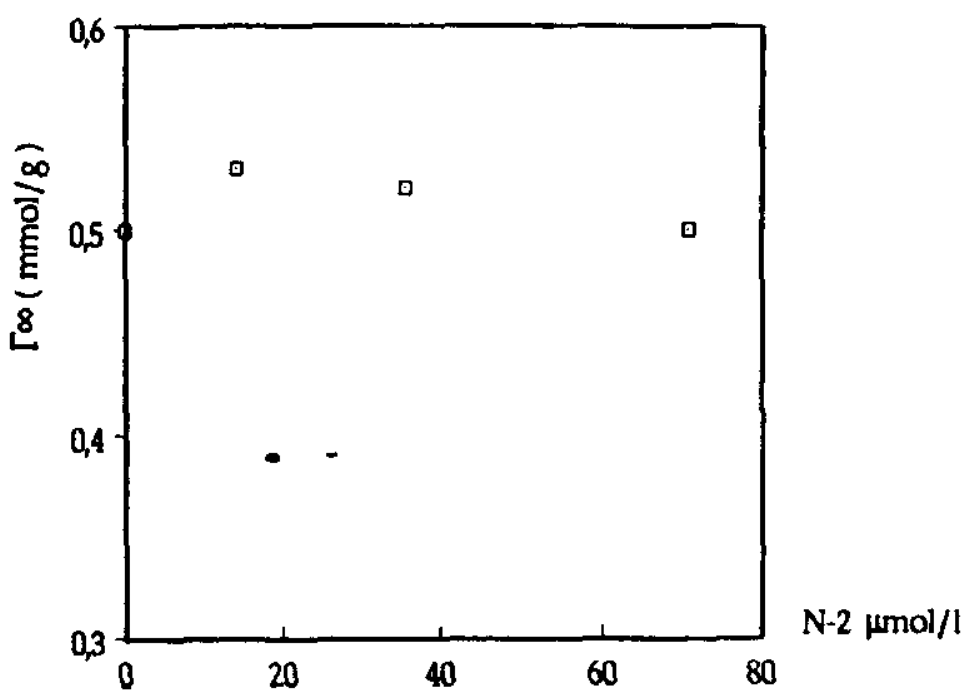

Figure 7 Évolution de la capacité maximale d'adsorption du DSS sur le CAP en présence du $\mathrm{N}-2 . \mathrm{C}_{\mathrm{DOS}}=50 \mu \mathrm{mol} / \mathrm{l}$.

Evolution of maximal adsorption capacity of SDS onto PAC with the presence $\mathrm{N}-2 . \mathrm{C}_{\mathrm{SDS}}=50 \mu \mathrm{mol} / \mathrm{.}$.

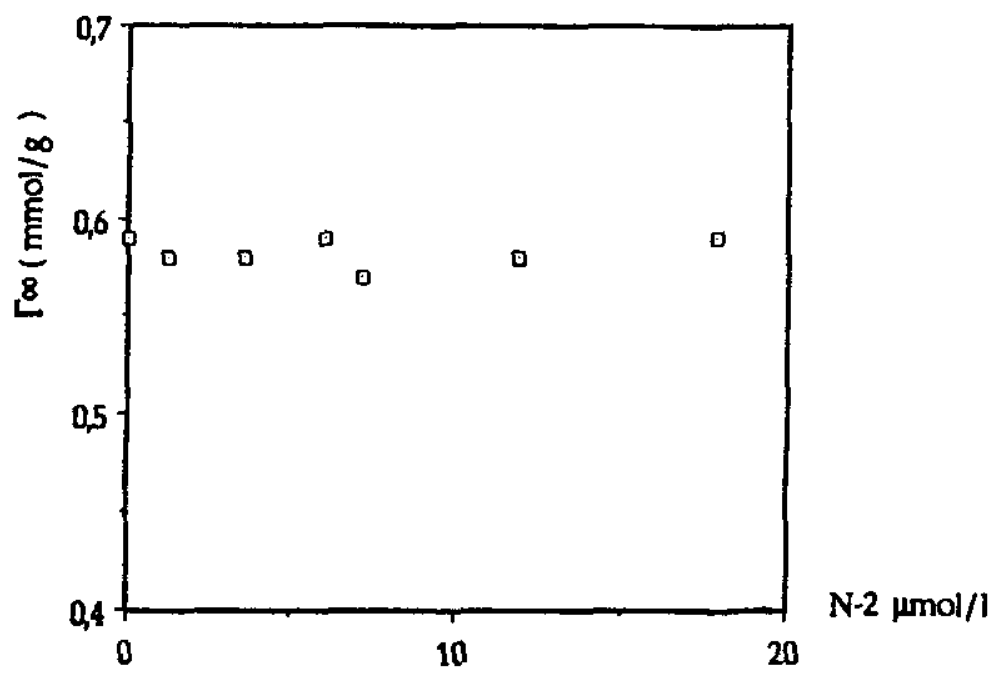

Figure 8 Évolution de la capacité maximale d'adsorption du BBDDA sur le CAP en présence du $\mathrm{N}-2$. $\mathrm{C}_{\mathrm{BBDDA}}=3,12 \mu \mathrm{mol} / \mathrm{l}$.

Evolution of maximal adsorption capacity of $B D D A B$ onto $P A C$ with the presence $\mathrm{N}-2$. $C_{B D D A B}=3,12 \mu \mathrm{molh}$. 


\section{4 - CONCLUSION}

L'adsorbabilité des composés organiques et leurs interactions au cours de l'adsorption avec d'autres composés (coadsorbats) constituent des facteurs déterminants pour prédire et évaluer les performances d'un matériau adsorbant lorsqu'il est en présence d'un mélange de ces composés.

Afin de mieux comprendre l'efficacité d'élimination, et les interactions entre les composés (BBDDA, DSS, N-2), nous avons étudié ladsorption du mélange (DSS $+\mathrm{N}-2)$ et $(B B D D A+N-2)$. Ces résultats permettent d'examiner l'effet de l'ordre d'introduction des co-adsorbats sur l'élimination des différents composés étudiés.

Les résultats expérimentaux montrent que la présence du $\mathrm{N}-2$ inhibe en partie l'adsorption du DSS. Lorsque le mélange (DSS + CAP) est en équilibre d'adsorption, l'ajout du N-2 provoque la désorption d'une fraction de DSS adsorbé et le nouvel équilibre établi correspond à celui obtenu pour les mêmes concentrations en solutés, lorsque ceux-ci sont introduits simultanément. Les résultats montrent que quel que soit l'ordre d'introduction des solutés, les concentrations en DSS à l'équilibre sont sensiblement égales. Le même comportement a été remarqué avec le $\mathrm{N}-2$ lequel voit son adsorption inhibée en partie par le DSS.

Lorsque le DSS est ajouté au mélange (N-2 + CAP), il provoque la désorption de celui-ci, par contre l'ajout du N-2 au mélange DSS-CAP laisse la concentration résiduelle en co-adsorbat sensiblement identique à celle obtenue lorsque le N-2 et DSS sont introduits simultanément.

Les résultats obtenus pour le tracé des isothermes de LANGMUIR dans le cas de l'adsorption du N-2 et BBDDA pour différents ordres d'introduction montrent que l'ordre d'introduction des co-adsorbats intervient tout en faisant diminuer la valeur de la constante d'équilibre d'adsorption de l'un des deux solutés $\mathrm{N}-2$ et BBDDA et que la capacité maximale d'adsorption $\mathrm{G}_{\infty}$ du BBDDA reste constante quel que soient l'ordre d'introduction et la concentration du co-adsorbat dans le domaine des concentrations étudiées.

La capacité maximale d'adsorption $\Gamma^{\circ}$ du $\mathrm{N}-2$ dans le système $(\mathrm{N}-2+\mathrm{CAP}$ + BBDDA) passe de 1,67 à 1,5 mmol/g dans les systèmes ( $\mathrm{N}-2$ + BBDDA + CAP) simultanément ou dans le système (BBDDA + CAP) $(3 h)+\mathrm{N}-2$; par contre la capacité maximale $\Gamma^{\infty}$ du $\mathrm{N}-2$ diminue fortement lorsque la concentration du BBDDA croît.

L'ajout du $\mathrm{N}-2$ après 3 heures de contact du BBDDAVCAP ne provoque pas la désorption de ce dernier (BBDDA); le même comportement a été observé avec le $\mathrm{N}-2$ quand il est introduit dans un premier temps et que le BBDDA est introduit après 3 heures de temps de contact entre le $\mathrm{N}-2$ et le CAP. 


\section{RÉFÉRENCES BIBLIOGRAPHIQUES}

DORE M., SOHM B., LEGUBE B., et BRUNET $R_{1}$ (1982), Effect of ozone on adsorbability and biodegradability of a few organic compounds over granular activated carbon. Env. Technol. Lett., 3, 375-384

DUSART O., MAZET M., ALLALI-HASSANI M., SOUABI S., YAACOUBI A., et CASTEIGNAU M, (1988), Now methods to evaluate micromolar quantity of anionic, cationic and non ionic surfactants in water. Congrés mondial des agents de surface. Paris 24-27 mai

DUSART O., MAZET M., KHADIRIAN R. D et ALLALI- HASSANI M. (1985), Adsorption des tensioactifs non ioniques en quantités micromolaires sur charbon activéadsorption mixte anionique-non ionique. $J$. Chim. Phys. Fr. 82, (10) 983-991

GAID K., CAVELIER C., et MARTIN G., (1982), Wat Res.16 (1), 7-17
LAFRANCE P., MAZET M., et VILLESSOT D., (1985), Wat. Res. 19,1059-1064

LANGMUIR I, (1915), J. Chem. Soc. 37, 1139

LE CLOIREC P., et MARTIN G, (1984), Le charbon actif le matériau et son utilisation dans le traitement de l'eau. T.M.S L'eau, 4 , 175-182

MAZET M., YAACOUBI A., LAFRANCE $P$, (1988), Wat. Res. 22, 132

PREVOST M., DESJARDINS R. LAFRANCE $P,(1990)$, Bilan sur le traitement biologique de l'eau potable. Sciences et Techniques de l'Eau. 311-319

SHELTON R.S., VAN CAMPEN M.G., TILFORD C.J., LANG, H.C., NISONGER L., BENDELIN F.J. et RUBENKOENIG H.C, (1948), J. Am. Chem. Soc. 68, 753-755 